\title{
Wave propagation phenomena in case of clamp-on ultrasonic systems applied on liquid filled pipes
}

\author{
Wöckel, Sebastian; Hempel, Ulrike; Auge, Jörg \\ Ifak - Institut für Automation und Kommunikation e.V. Magdeburg / Measuring \& Analyser Systems \\ Werner-Heisenberg-Str. 1, 39106 Magdeburg
}

\section{Motivation}

Ultrasonic measurements are common techniques in industrial liquid analysis for level detection or monitoring the concentration, the distribution of particles or the flow velocity in pipes [1]. Especially the noninvasive clamp-on configuration is an interesting approach due to its non-destructive nature and easy handling. Such a sensor can be mounted on existing reactors or pipes without either stopping the process or rebuilding the system. However, such a technique often needs a sophisticated signal analysis in order to discriminate the measurement effect from disturbances caused by the multimodal sound propagation. The disturbing signals occur from wave propagation within the metallic or plastic pipe wall, the ringing due to low damping and from mode conversions at the solid-liquid-interfaces. Up to now the multiple reflections and the wave travelling in the pipe wall are handled by optimizing the sensor position with empirical calibration, only. However, in cases where the sensors position can not be varied and the multimodal sound propagation is not known, the signal containing the medium information can not be extracted without a media dependent signal pre-processing. Precisely it can be superposed by the pipe wall waves, which sometimes are reduced with damping mats [2], and the focusing wave occurring due to mode conversion at the curvilinear inner pipes interface towards the liquid. Especially the disturbing mode converted waves travelling at the solid liquid interface depend on the properties of the liquid media. Hence, all systems have in common, that they do not handle multiphase applications because of the changing propagation conditions within the pie (varying acoustic impedances along the acoustic path). As consequence the clamp-on liquid analysis is carried out for a few cases only, e.g. for concentration measurements of homogeneous media or homogeneous distributed particles [2]. Nonetheless, all systems need a calibration of sensor position and time windowing.

To enhance the performance and reliability of clamp-on ultrasonic applications towards analysis tasks [3], a sophisticated signal analysis [4] and knowledge of the wave behaviour, the mode conversion and the influence of dispersion within the large scale complex technical structure with a high precision is required. In order to design such a robust sensor system a tool is needed that estimates the behaviour of multimodal sound propagation within a pipe prior to the sensor setup and measurement process. The preestimation of the sound phenomena based on the aforementioned techniques could save engineering time and costs compared to conventional and empirical calibrations. Accordingly this paper discusses the multimodal sound wave propagation in small up to large scale pipes filled with multiphase liquid systems. A first estimation of all expected wave propagation paths, corresponding travel time and multiple reflections is performed using the Mason-theory [5]. It will be shown that this linear modelling is adequate to estimate the travel time with certain precision for optimizing the sensors position or choosing proper time windows in the acquired ultrasonic signals where no significant disturbing effects occur. The paper further presents simulations in combination with experimental studies that are used to investigate the geometric aspect of the mode conversion. Exemplarily a clamp-on configuration with varying sensor position, different wideband excitation in the range of $f=[0.2,0.5,2.25] \mathrm{MHz}$, the transmission, the reflection from a liquid interface and the disturbing signals are discussed.

\section{Linear model}

\subsection{Modelling of the multimodal wave propagation}

Exemplifying the clamp-on liquid analysis a circular pipe was chosen that refers to common analysis tasks in industry. Regarding the main system draft of a pipe cross section filled with a homogeneous medium (Figure $1 \mathrm{a}$ ) and assuming a lateral dimension of the material under test (MUT) larger than the diameter of the transducers $\left(d_{\text {transducer }}\right)$ the transmission can be reduced to a one dimensional model with layer thicknesses $d_{i}$ (Figure $1 \mathrm{~b}$ ). This modelling is valid since a narrow radiation pattern $F_{\text {real }}(\varphi)[6]$ in boresight direction of the wideband transducers can be assumed. Due to that simplification a signal oriented model can be used to describe the propagation of ultrasonic waves and the medium and pipe dependent measurement effects. In Figure $1 \mathrm{~b}$ all signal paths between the transmitter and the receiver are represented by a Mason-graph [5]. 


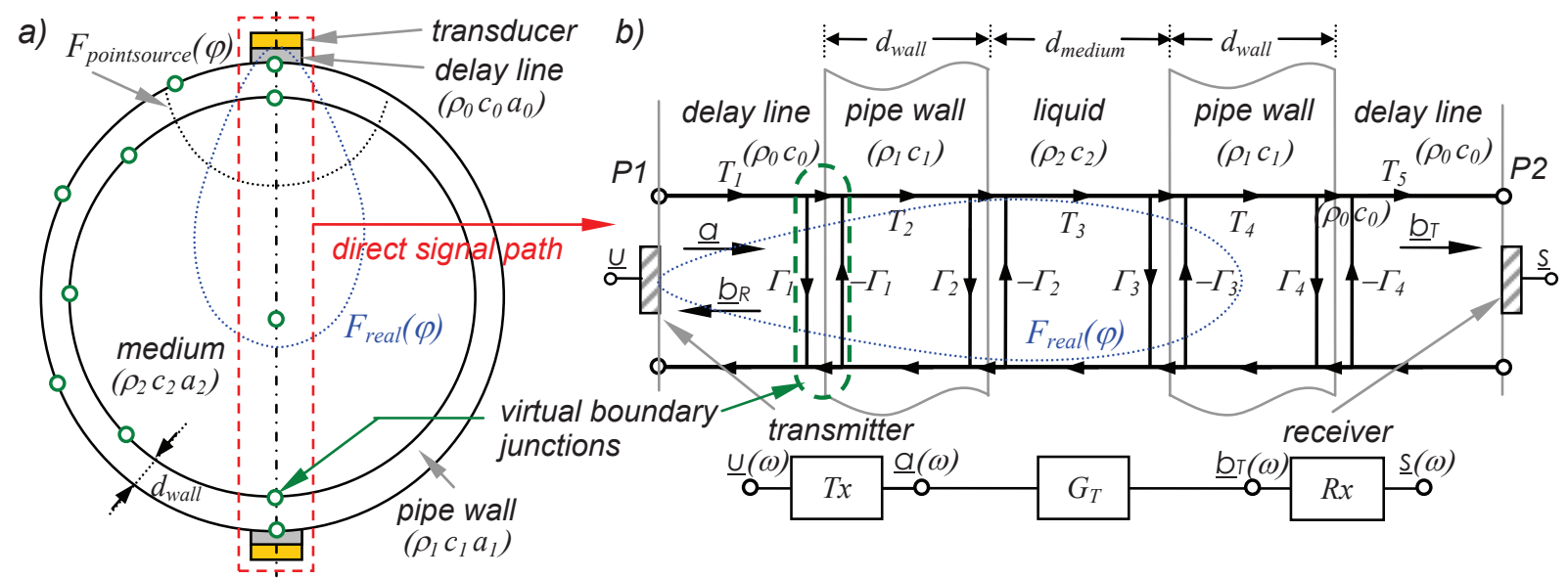

Figure 1: a) Draft of the measurement setup: pipe cross section with ultrasonic transducers (V323-SU $f=2.25 \mathrm{MHz}$ $B=2 \mathrm{MHz}$ ) in clamp-on configuration and reduced linear model for multimodal wave propagation and mode conversion at interface boundaries, b) layer system in boresight direction and corresponding linear model for wideband transducer with narrow radiation pattern $F_{\text {real }}(\varphi)$ and directivity $>>1$, describing the wave propagation within the layered structure and assuming no mode conversion with perpendicular incident angle

This model is based on the linear wave propagation. It includes the impinging $a(\omega)$, transmitted $b_{T}(\omega)$ and reflected $b_{R}(t)$ waves, the coefficients of the physical transmission $\left(T_{i}\right)$ within the layers and the reflection $\left(\Gamma_{i}\right)$ at the acoustic interfaces and the transformation of the signal by the transducers. Based on that Mason-model an analytical solution can be derived to calculate either the transmitted or the reflected signal. In Figure $2 a(\omega)$ and $b_{i}(\omega)$ represent normalized pressure waves in frequency domain at the excitation reference plane and the measurement plane. The propagation paths within the media are approximated by complex transmission function $T_{i}(\omega)(1)$.

$$
T_{i}(\omega) \approx T(\omega) \cdot e^{-j \omega \tau(\omega)} e^{j \sigma}
$$

Where $T$ is the real transmission factor, $\omega=2 \pi f$ - angular frequency, $\tau$ - time of flight and $\sigma=\alpha_{S}+\alpha$ an effective divergence loss $\left(\alpha_{S}\right)$ and damping $(\alpha)$ factor. The planar wave behaviour at the interfaces with different acoustic impedance $Z_{i}$ is represented by complex reflection coefficient $\Gamma_{i}(\omega)$ as well.

$$
\Gamma_{i}=\frac{Z_{i+1}-Z_{i}}{Z_{i+1}+Z_{i}} \quad \text { with } \quad Z_{i}=\rho_{i} c_{i}
$$

Considering all paths in the graph for a homogeneous medium an analytical transfer function $G_{T}$ can be derived $[7,8]$, which describes the wave propagation from transmitter $P 1$ to receiver $P 2(3)$.

$$
G_{T}=\frac{b_{T}}{a}=\frac{1}{\Delta} T_{1} \sqrt{1-\Gamma_{1}^{2}} T_{2} \sqrt{1-\Gamma_{2}^{2}} T_{3} \sqrt{1-\Gamma_{3}^{2}} T_{4} \sqrt{1-\Gamma_{4}^{2}} T_{5}
$$

with $\quad \Delta=1+T_{2}^{2} \Gamma_{1} \Gamma_{2}+T_{3}^{2} \Gamma_{2} \Gamma_{3}+T_{4}^{2} \Gamma_{3} \Gamma_{4}+T_{2}^{2} T_{3}^{2} \Gamma_{1} \Gamma_{3}+T_{3}^{2} T_{4}^{2} \Gamma_{2} \Gamma_{4}+T_{2}^{2} T_{3}^{2} T_{4}^{2} \Gamma_{1} \Gamma_{4}+T_{2}^{2} T_{4}^{2} \Gamma_{1} \Gamma_{2} \Gamma_{3} \Gamma_{4}$

The transformation of the waves at the reference planes $P_{i}$ by the (piezoceramic) transducers are described with the coupled transfer $(T x)$ and receive $(R x)$ functions which map a scalar value (voltage signal $u(t)$ and $s(t))$ on vectorial wave fields (pressure $\left.a(t), b_{T}(t)\right)$.

$$
a(\omega)=T x(\omega) \cdot \underline{u}(\omega) \quad \underline{s}(\omega)=R x(\omega) \cdot b_{T}(\omega)
$$

Omitting multiple reflections at the transducers surface, which are already included in the graph, the received transfer signal $\underline{s}(\omega)$ can be calculated in frequency domain with:

$$
\underline{s}(\omega)=R x(\omega) \cdot G_{T}(\omega) \cdot T x(\omega) \cdot \underline{u}(\omega)
$$

This received signal $\underline{s}(\omega)$ represents the impulse response function (IRF) of the whole system which contains the information on the MUT. In order to classify the MUT (characterizing the unknown medium) by its transmission signal one has to extract the transfer function $G_{T}$. Concerning equ. (5) $G_{T}$ is disguised by the IRF of the transducers $\left(R x^{*} T x\right)$ and the transmitted signal $\underline{u}(\omega)$ [9]. Including a number of disturbing components the total measurable electrical signal $s_{M E A S}$ becomes:

$$
s_{\text {MEAS }}=s_{r}+s_{\varphi}+s_{X T}+s_{B K}+n+r+c
$$

$s_{r}$ direct transmission signal in $r$-direction (see equ. 5), $s_{\varphi}$ - transmission along the pipe wall in $\varphi$-direction Scattering on irregular and uneven interfaces as well as particles are treated as additional disturbances (clutter $c$ ). Compared to clutter, the noise $n$ will only cause minor effects. Other perturbing components can be removed by time gating of short non-overlapping signals (typical examples: cross talk $s_{X T}$ and multiple reflections $r$ ) and subtraction of static background reflections $s_{B K}$. In the present case, the back- 
ground removal is a challenging task since the MUT may be located within reactors with complex geometry. In that case the wave propagation in boresight direction $\left(s_{r}\right)$, in angular direction on the pipe wall $\left(s_{\varphi}\right)$ as well as mode conversion due to boundary effects have to be known precisely to separate the targets' signal from reflection and creeping signals of the reactors housing. Equation 6 describes a simple model regarding all scattering effects to be independent from each other. The validity of this assumption is limited but should be adequate to extract $s_{r}(\omega)$ and $s_{\varphi}(\omega)$ out of the total measured signal $s_{M E A S}(\omega)$.

The direct transmission path model (Figure $1 \mathrm{~b}$ ) assumes perpendicular incident neglecting mode conversion, divergence loss and lens effects of the curvilinear pipe, which is valid for optimized wideband transducers only. Concerning varying incident angle, which is caused by edge waves or ideal point source with a radiation pattern $F(\varphi)=1$, the signal graph (Figure $1 \mathrm{~b}$ ) has to be expanded with additional signal path in angular direction including junctions at the boundaries (Figure $1 \mathrm{a}$ ). These junctions are similar to a macroscopic discretization of the solution domain (cross section). Further the scalar transmission and reflection functions $T, R$ have to be replaced with tensors $\underline{\underline{T}} \underline{\underline{R}}$ (mode conversion matrix) including the angle dependent mode conversion between longitudinal and transversal waves and superposition of multimodal sound transmission. Exemplarily the initial junction becomes

$$
\underline{\underline{T}} \cdot \underline{a}=\left[\begin{array}{ll}
T_{L L} & T_{L T r} \\
T_{T r L} & T_{T r T r}
\end{array}\right] \cdot[F(\varphi)] \cdot\left[\begin{array}{l}
a_{L} \\
a_{T r}
\end{array}\right]=\left[\begin{array}{l}
b_{L} \\
b_{T r}
\end{array}\right],
$$

where the impinging signal a has to be weighted with the radiation function $F(\varphi)$.

\subsection{Verification of analytical model}

The equation (5) describes the unimodal wave propagation (longitudinal) in boresight direction and neglects all mode conversion due to non perpendicular incident angle. This modelling has been verified with the ultrasonic transmission on a metallic steel pipe filled with water (Table 1). Corresponding to the draft in Figure 1 two ultrasonic transducers (V323-SU Panametrics) are placed in transmission setup via a delay line on the surface of the steel pipe. In the measurements a pulse excitation with a centre frequency of $f=2.25 \mathrm{MHz}$, a $10 \mathrm{~dB}$-bandwidth of $B=2 \mathrm{MHz}$ and a sampling frequency of $f_{T}=50 \mathrm{MHz}$ was used.

a)

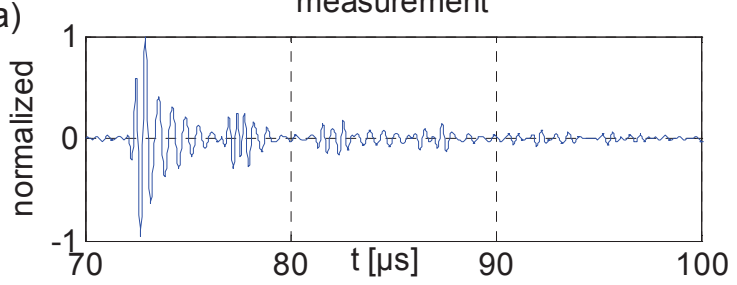

non-divergent prediction (envelope)

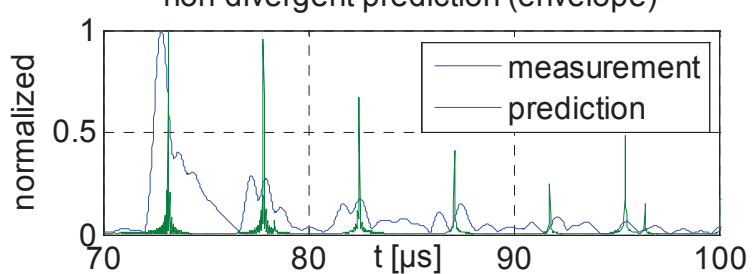

b)
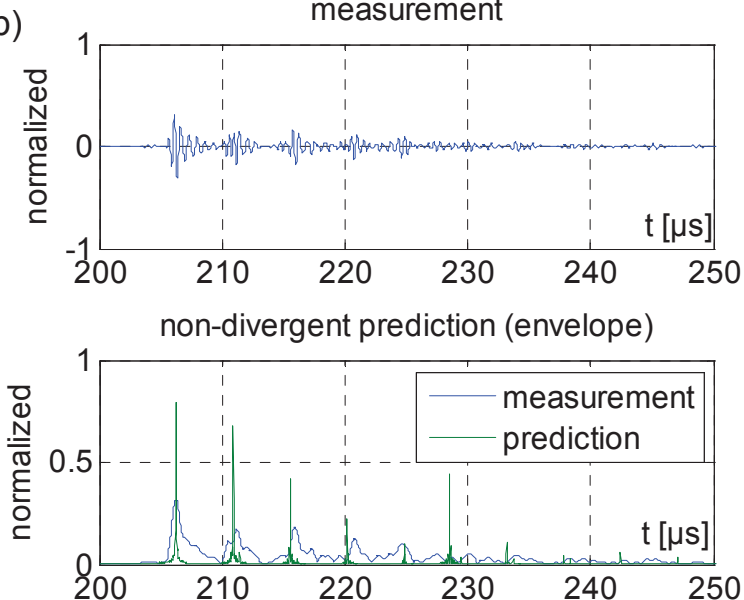

Figure 2: Model solution and measurement of transmitted signal $s(t)$ with $d_{\text {medium }}=100 \mathrm{~mm}, d_{\text {wall }}=13 \mathrm{~mm}, d_{\text {transducer }}$ $=6 \mathrm{~mm}$, and material parameter corresponding to table 1; neglecting mode conversion, divergence and lens effect: a) first time arrival, b) first multiple reflection

Figure 2 shows the raw signal of first time arrival (a) and first multiple reflection (b) of a transmission through the pipe walls with water as intermediate layer in comparison to the corresponding model result $\left(2^{\text {nd }}\right.$ row $)$. The amplitudes were normalized to the full scale range of the receivers' amplifier. The model signal $G_{T}(5)$ displays the position (centre of gravity [9]) and weight of predicted signals only. Note, that the measurable signal $s(t)$ is an convolution of $G_{T}$ with the transducers transmissions function $T X+R x(5)$, which will lead to a spreading of the signal trace. Comparing $G_{T}$ with the envelope of the measured signal, the travel time of the longitudinal first time arrival, the multiple ringing in the pipe wall and the first multiple reflection can be estimated with a precision of $>90 \%$. Hence based on that data proper time windows can be chosen for time gating. Here the time deviation between the measurement and the model solution is affected by a thermal drift in wave speed and uneven pipe wall thickness. The variation of the weights corresponds to the neglection of the divergent wave front and focusing effects of the curvilinear pipe wall. However the measurements in addition to calculations proof that in case of perpendicular incident angle, narrow radiation pattern and homogeneous media (even multiphase media) neither crucial disturbing waves caused by transversal mode conversions nor angular signal path appear. 


\section{Simulation of two-dimensional sound propagation}

The simulation studies described here are used to investigate the sound propagation in case a divergent radiation pattern and edge waves of the transducers have to be assumed. Following based on the results the main parameters, which need to be additionally included in the linear model, are exposed with evaluation of their importance on the multimodal sound propagation.

\subsection{Homogeneous media}

In addition to the linear modelling a simulation of the two-dimensional sound propagation with finite elements was supposed to reveal the multimodal wave behaviour of single longitudinal modes influenced by the geometry only. The propagation of a differentiated Gaussian pulse with low frequency bandwidth of $f$ $=200 \mathrm{kHz}$ and $500 \mathrm{kHz}$, where edge waves and low directivity are expected, was regarded within a circular pipe. Figure 4 exemplarily shows snapshots of a transient multimodal wave travelling through a water filled acrylic pipe in case of an impinging impulse excited by a clamp-on sensor on the outer pipe wall. Even for that idealised configuration beside the direct wave (Figure 3 a) additional waves travelling inside the pipe (c) as well as multiple reflections (d) and focusing effects (b, e) caused by curvilinear boundaries are obvious. In particular the quasi longitudinal wave (c), which is travelling along the pipe wall delivers both a structure born wave (concentrating on the sound hard boundary - see section 3.3) and a "mode"converted wave (b) in the media. One can see that the transducer on the circumference of the pipe will receive different complex signals, whose characterization e.g. for analysing the unknown liquid cannot be provided without secondary information from calibration, simulation studies or enhanced modelling.

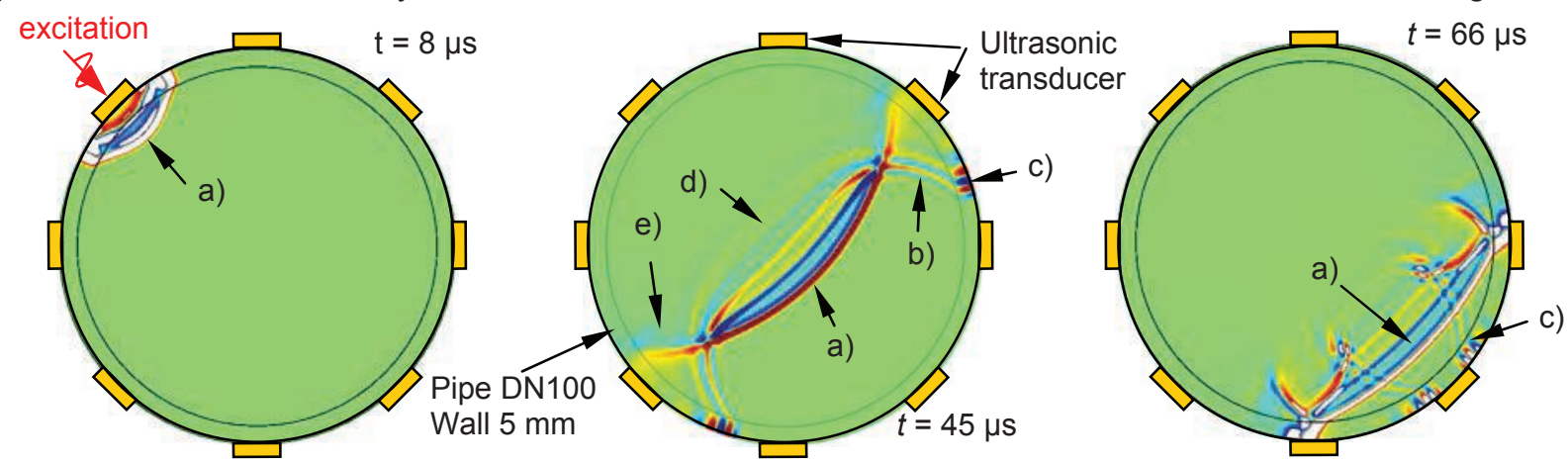

Figure 3: Clamp-on sensor configuration on a pipe (DN100) and snapshot of ultrasonic pressure wave field with pulse excitation (differential gauss pulse with $f_{0}=200 \mathrm{kHz}$ bandwidth): a) direct wave, b) converted wave, c) quasi longitudeianl "surface" wave within pipe wall, d) pipe wall reflection, e) focus wave. Parameter refer to Table 1.

\subsection{Multiphase media}

Considering a simple application of monitoring a filling level or phase boundary with a reflection configuration a more complex two-phase system instead of a homogeneous one has to be regarded (Figure 4 a). The measurable signal at receiver (R2) consists of multiple impulses where the interesting information ( 2 . boundary reflection) is formed by a small part only. As like the transmission signal in the homogeneous case (Figure 3 ) the disturbing signals $(1 ., 4$.) can totally overlap and mask the boundary reflection for disadvantageous ratio of sound velocity, pipe dimensions and sensor positioning. Further the speed and amplitude of disturbing signals along the pipe vary caused by changing acoustic loading at the inner wall.

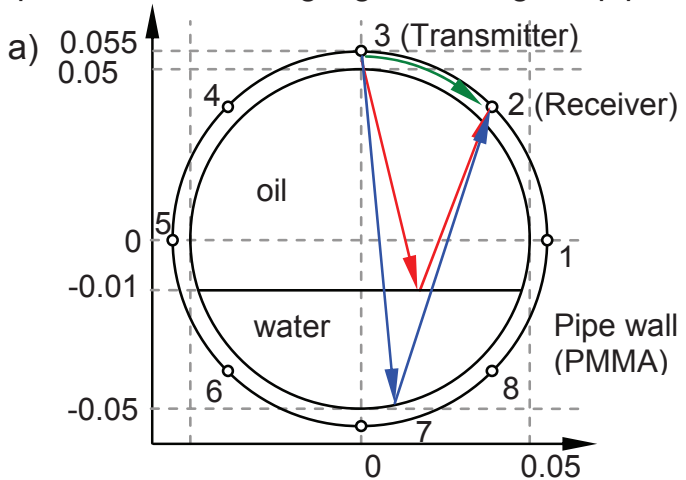

b)

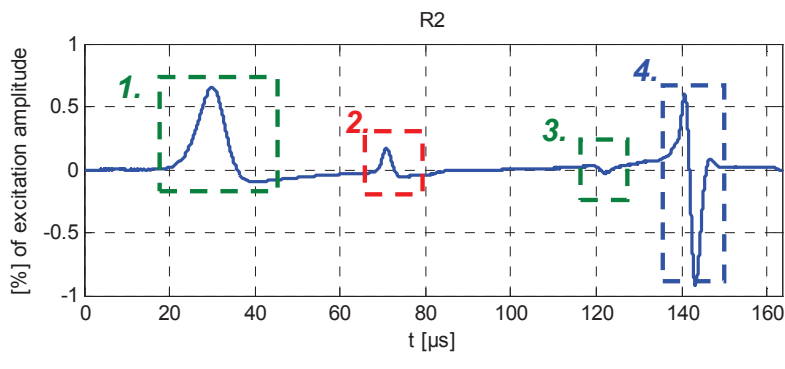

Figure 4: Reflection configuration assuming a point source (T3) in case of a pipe filled with a two-phase system: a) draft of cross section ( $d_{i}=100 \mathrm{~mm}$ diameter, $d=5 \mathrm{~mm}$ wall thickness, 8 sensor points), b) data in receiver point 2 for a water level of $h=40 \mathrm{~mm}$ (excitation: differential gauss pulse with $f_{0}=200 \mathrm{kHz}$ bandwidth normalized to amplitude in pipes wall). The main propagation paths and the corresponding receiver signals are marked: 1 . wave travelling in pipe wall, 2. reflection from media interface, 3. multiple reflection and 4. reflection at inner pipe wall. (refer Table 1) 
The sonogram of intensity plots in Figure 5 exemplifies the measurable signal in point R2 for common source point T3 for consecutive rising filling level of water $h=10 \ldots 90 \mathrm{~mm}$. In the left diagram the raw data with labelling of the perturbing wave within (1.) and the reflection (4.) at the pipe wall as well as the boundary reflection (2.) and the sample trace from Figure $4 \mathrm{~b}$ are shown. Depending on the sensor configuration some filling levels can not be characterized unless the overlapping signals are removed so that the interesting reflection signal gets obvious (Figure $5 \mathrm{~b}$ ). Especially in the present example the reflection for a high filling level of $h>80 \mathrm{~mm}$ as well as the multiple reflection signals (3.) can be detected not until background subtraction.

a)

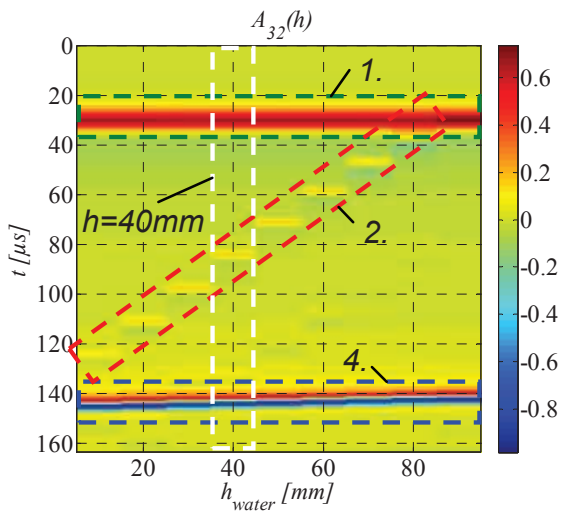

b)

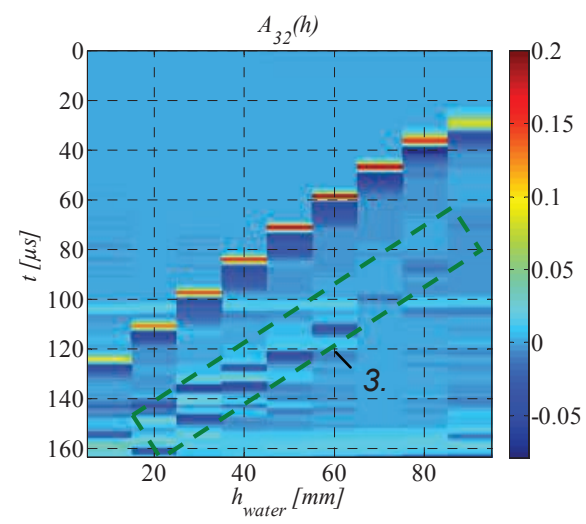

Figure 5: Simulation: a) sonogram of raw signals $A_{32}(h)$ from clamp-on sensor 3 to 2 with rising water level and perturbing signals (Figure 4), b) sonogram after background subtraction with remaining interface reflection and the multiple reflection.

\subsection{Influence of pipe wall}

The geometry and acoustic property of the pipe has an essential influence on the sound propagation. Dependent on the ratio of the sound velocities in relation to the spatial dimensions overlapping and superposition of different wave phenomena and mode conversions at the inner pipe surface towards the medium may occur. Here the main effects are exemplified by comparing simulations of the configuration from (Figure 4 a) with infinite (impedance) and finite (reflecting) boundary conditions. Figure 6 and Table 1 include the signal path and the analytical travel time of the longitudinal waves $\tau_{i}$ and the input parameter of the material. For the sake of briefness the signals for an acrylic (PMMA) pipe are discussed only. Here the sound-reflecting (sound hard) boundary condition approximates the real case of a clamp-onmeasurement. With the two-dimensional simulation the appearance of quasi longitudinal "surface" waves, the focus waves (mode conversion) at the inner pipe wall and the travel time can be reproduced (Figure 7). The acoustic emission towards air is negligible.

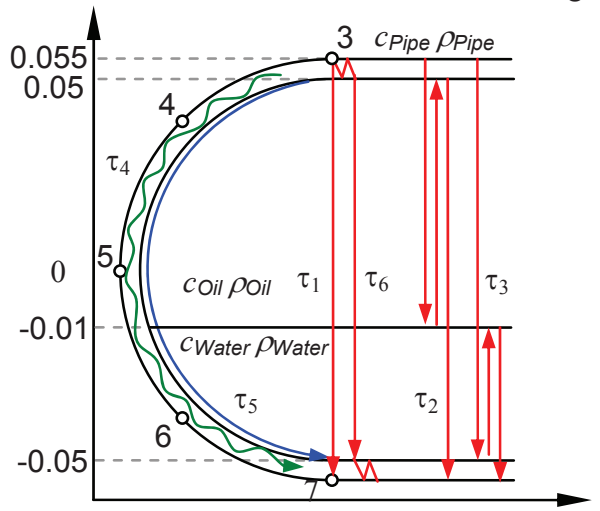

Table 1:

parameter of material

$c$ Water $=1480 \mathrm{~m} / \mathrm{s}$

$\rho_{\text {Water }}=998 \mathrm{~kg} / \mathrm{m}^{3}$

$c_{\text {Oil }}=1445 \mathrm{~m} / \mathrm{s}$

$\rho_{\text {Oil }}=912 \mathrm{~kg} / \mathrm{m}^{3}$

$c_{P M M A}=2567 \mathrm{~m} / \mathrm{s}$

$\rho_{P M M A}=1190 \mathrm{~kg} / \mathrm{m}^{3}$

$c_{\text {Steel }}=5920 \mathrm{~m} / \mathrm{s}$

$\rho_{\text {Steel }}=7800 \mathrm{~kg} / \mathrm{m}^{3}$

\begin{tabular}{|r|r|r|}
\hline \multicolumn{3}{|c|}{ travel time } \\
\hline & PMMA & Steel \\
\hline delay & $+4 \mu \mathrm{s}$ & $+4 \mu \mathrm{s}$ \\
\hline$\tau_{1}$ & $72,45 \mu \mathrm{s}$ & $70,24 \mu \mathrm{s}$ \\
\hline$\tau_{2}$ & $155,49 \mu \mathrm{s}$ & $153,28 \mu \mathrm{s}$ \\
\hline$\tau_{3}$ & $126,50 \mu \mathrm{s}$ & $124,30 \mu \mathrm{s}$ \\
\hline$\tau_{4}$ & $64,25 \mu \mathrm{s}$ & $29,20 \mu \mathrm{s}$ \\
\hline$\tau_{5}$ & $106,00 \mu \mathrm{s}$ & $67,66 \mu \mathrm{s}$ \\
\hline$\tau_{6}$ & $\tau_{1}+11 \mu \mathrm{s}$ & $\tau_{1}+5 \mu \mathrm{s}$ \\
\hline
\end{tabular}

Figure 6: Draft of pipe cross section and a water-oil two phase system with illustration and estimation of travel time for longitudinal wave propagation: $\tau_{1}$ - direct wave, $\tau_{2}, \tau_{3}-$ multiple reflection, $\tau_{4}$ - quasi longitudinal "surface" wave, $\tau_{5}$ - focused conversion wave at inner pipe wall, $\tau_{6}$ - ringing in pipe wall

As one can see in Figure 7 the main transmission signal $\left(\tau_{1}\right)$ is not influenced by the boundary condition. In contrast the quasi longitudinal "surface" wave in angular $\varphi$-direction $\left(\tau_{4}\right)$ and constructive multiple reflection in radial $r$-direction $\left(\tau_{6}\right)$ vary due to changing boundary condition and pipe wall thickness. These effects are forced with an excitation frequency of $f=500 \mathrm{kHz}$ where the wavelength in PMMA equals a multiple integer of the pipe wall thickness. Hence the fast quasi longitudinal wave in the pipe wall is distinctive and appears prior the expected direct signal (first time arrival). With non multiple integer frequencies like $f=200 \mathrm{kHz}$ these waves are reduced. Furthermore varying impedance in case of layered multi- 
phase systems lead to changing of the behaviour of the focused conversion wave at the inner pipe diameter. Hence an additional signal dependent of filling level occurs $\left(\tau_{5}\right)$.

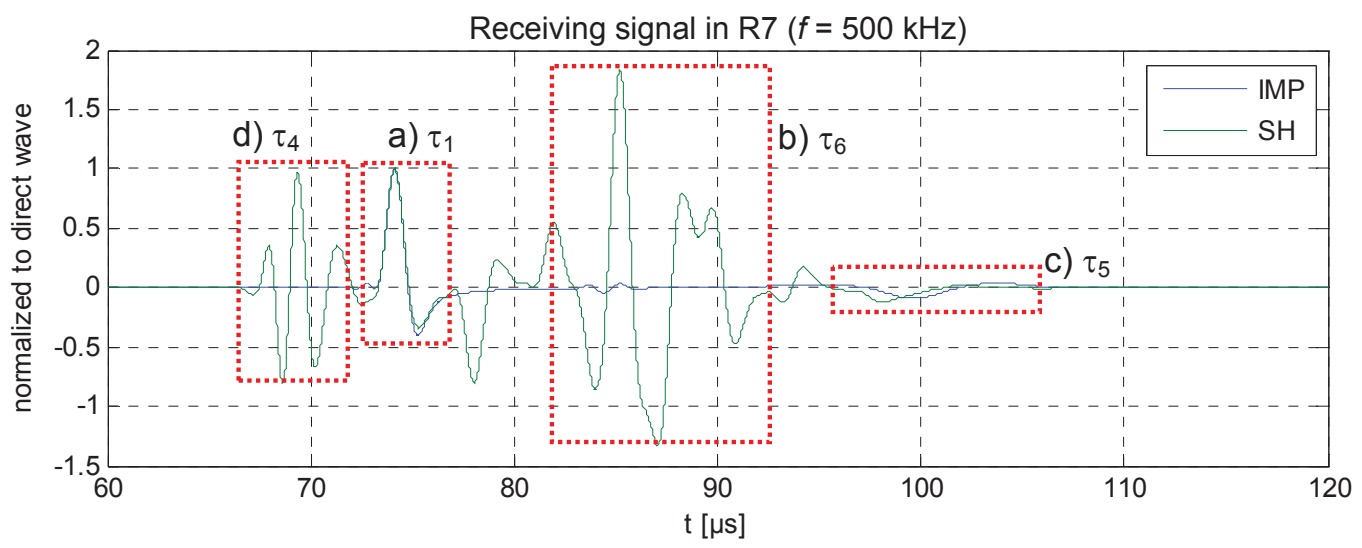

Figure 7: Transmission signal $(f=500 \mathrm{kHz})$ in receiver point $\mathrm{R} 7$ with common source point T3 in case of reflecting (SH-sound hard) and matched (IMP-impedance) boundary conditions of an acrylic pipe: a) direct wave (interesting signal), b) ringing (multiple constructive reflection) due to pipe wall thickness, c) conversion wave in media at inner pipe wall, d) quasi longitudinal wave in pipe wall (refer Table 1)

\section{Conclusion}

When a high directivity transducer can be assumed in most cases the linear modelling is adequate to describe the transmission effects in case of clamp-on transducers. On one side the time window can be estimated independent from temperature drift and on the other multiple reflections can be removed even if they overlap with the direct signal. Even for lower frequency range the disturbing signals are mainly caused by quasi longitudinal wave phenomena in the pipe wall and are not mainly due to transversal or structure-borne mode conversion. In many applications, if no explicit point source radiation pattern and edge waves need to be assumed, the linear modelling suffices to provide an enhanced signal analysis. Here the ultrasonic frequency in relation to the pipe wall thickness, the directivity and the boundary condition are crucial for the disturbing effects. Further the adaptation of the excitation wavelength (frequency) to non multiple integer of the pipe wall thickness can additionally reduce the disturbing effects. Most of the disturbing signals are caused by the pipe wall outer surface. The corresponding disturbing boundary effects (ringing $\tau_{6}$, quasi longitudinal wave $\tau_{4}$ ) do not occur if a matched or damped boundary can be provided (Figure 7 comparison between sound hard and impedance boundary). Thus, to provide such impedance matched conditions some applications use damping mats at the pipe surface. Ringing in pipe wall stretches the impinging signal. Hence the relation between wavelength and pipe wall thickness becomes important for spatial resolution also. Dependent on size and frequency especially for low frequency applications the direct signal and the pipe wall ringing may overlap masking the wanted signal. For that, the linear modelling, as exemplified, can be used to remove the pipe wall ringing [7]. The modelling enables: the adaptation of the ultrasonic frequency and the sensor position to pipe geometry and material and the signal oriented time gating for optimal separation of an interesting signal and disturbances. Neither additional constructive changes nor time expensive calibration need to be performed.

\section{Literature}

[1] M. Panicke; „Diagnosis and Evaluation of Ultrasonic Clamp-on Measurements“, $8^{\text {th }}$ South East Asia Hydrocarbon Flow Measurement Workshop, Malaysia 2009.

[2] S. Schwidder, S. Machefer, K. Schnitzlein; „Konzentrationsmonitoring bei Gas/Flussig-Reaktionen mittels einer modifizierten Schallgeschwindigkeits-Sonde", CIT, vol. 80; number 3, pp. 389-392, 2008.

[3] M. H. Fazalul Rahiman, et. Al: „Modelling Non-Invasive Ultrasonic Tomography for Industrial Liquid/Gas Flow Application", Proc. Int. Conf. on Modeling, Simulation \& Visualization Methods (MSV'06)", 2006.

[4] P. Holstein, H.J. Münch (Sonotec GmbH): „Handling großer Datenmengen in der Schallemission - ein Beitrag zur Algorithmenentwicklung", DGZfP-Jahrestagung, 2009.

[5] S.J Mason: „Further Properties of Signal Flow Graphs“, Proc. Institute of Radio Engineers, vol. 44, number 7

[6] Kraukrämer: "Werkstoffrüfung mit Ultraschall", ISBN-10: 3540157549

[7] Wöckel, S., Hempel, U., Auge, J.: Phase boundary characterization in liquids by acoustic waves. Special Issue Measurement Science Technology, Meas. Sci. Technol. 20124013 (6pp)

[8] Hempel, U., Wöckel, S., Auge, J.: Ultraschallbasierte Informationsgewinnung in der Verfahrenstechnik. Chemieund Ingenieurtechnik 2010, 82, No.4, S. 491-502

[9] Wöckel, S.; Hempel, U.; Auge, J.: Tomographical monitoring of processes with multicomponent and multiphase fluid mixtures. EUROSENSORS XXII, 07.-10.09.2008, Dresden, Tagungsband auf CD, S. 761-764. 